\title{
Acute heart failure from a rapidly progressing large tumor in the right atrium
}

\author{
I-Hsin Chang ${ }^{1}$, Lian-Jie Lin ${ }^{2}$, Ren-Chieh Wu ${ }^{1,3, *}$
}

\author{
${ }^{1}$ Department of Emergency Medicine, \\ Hualien Tzu Chi Hospital, Buddhist Tzu \\ Chi Medical Foundation, 970 Hualien, \\ Taiwan \\ ${ }^{2}$ Department of Otorhinolaryngology, \\ Hualien Tzu Chi Hospital, Buddhist Tzu \\ Chi Medical Foundation, 970 Hualien, \\ Taiwan \\ ${ }^{3}$ Program in Pharmacology and \\ Toxicology, Tzu Chi University, 970 \\ Hualien, Taiwan
}

\begin{abstract}
The diagnosis and treatment of intracardiac mass are challenging for emergency physicians. Dyspnea is one of the most common chief complaints in ER among patients with rapidly progressed heart failure without significant medical history. Establishing such an unusual diagnosis based on dyspnea patients requires more evidence. We present a case of a patient who was diagnosed with a rapidly progressing right atrial mass.
\end{abstract}

\section{Keywords}

Cardiac mass; Right atrium tumor; Heart failure

*Correspondence

uliwu@ms56.hinet.net

(Ren-Chieh Wu)

\section{Introduction}

Intracardiac mass is a rarely encountered phenomenon in the emergency room. An intracardiac mass can be classified into thrombus and non-thrombus. The symptoms caused by a cardiac mass are varied and mimic pulmonary or cardiac conditions depending on the location of the mass rather than the pathology. Our patient presented with a rapid onset of heart failure and was diagnosed with a cardiac mass via computed tomography scan (CT) in the emergency department (ED). For unexplainable cases of heart failure, in-depth radiological studies are recommended to identify the etiology.

\section{Case report}

A 72-year-old woman with a history of diabetes mellitus and hypertension visited ED with a complaint of dyspnea for about one week. After a series of negative diagnoses on blood exams and electrocardiography, cardiac sonography (Fig. 1) raised suspicions of heart failure (N-terminal pro-B type natriuretic peptide, NT-BNP; $956 \mathrm{pg} / \mathrm{mL}$, normal value <125). The patient was discharged with a tentative diagnosis of suspected heart failure.

She was sent to the ED again two weeks later for progressed exertional dyspnea associated with dry cough and dizziness. She had no orthopnea, chest pain, or pitting edema, and had not suffered a trauma or undergone operation recently. Her vital signs were normal: body temperature, $36.4{ }^{\circ} \mathrm{C}$; heart rate, 76 beats $/ \mathrm{min}$; respiratory rate, 24 times/min; blood pressure 171/72 $\mathrm{mmHg}$; and $\mathrm{SpO} 2,92 \%$ while using nonrebreather-mask (15 L/min). Physical examination showed a clear breathing sound without a heart murmur. Laboratory examinations revealed metabolic acidosis, elevated lactate $(6.5 \mathrm{mmol} / \mathrm{L}$, normal value $0.5-2.2 \mathrm{mmol} / \mathrm{L})$, elevated $\mathrm{d}$ - dimer (3747.23 ng/mL, normal value $<499 \mathrm{ng} / \mathrm{mL}$ ), normal fibrinogen ( $293.8 \mathrm{mg} / \mathrm{dL}$, normal value $180-350 \mathrm{mg} / \mathrm{dL}$ ), elevated NT-BNP $(1247 \mathrm{pg} / \mathrm{mL})$, and dynamic cardiac troponin-I elevation $(114 \mathrm{pg} / \mathrm{mL}$ to $191 \mathrm{pg} / \mathrm{mL}$ in four hours, normal range $<17.5 \mathrm{pg} / \mathrm{mL}$ ). A contrast-enhanced CT scan of pulmonary arteries revealed a large non-enhancing mass about $67 \times 50 \times$ $63 \mathrm{~mm}$ in size near the right atrium extending up to the inferior vena cava and adjacent hepatic vein (Figs. 2,3,4). The patient was admitted to the cardiovascular intensive care unit (ICU) for further management.

Transesophageal echocardiography (TEE) performed after ICU admission showed a large heterogenous cauliflower-like mass, $4 \times 6 \mathrm{~cm}$ in size, with septated and cavitated lesion occupying the right atrial cavity, compressing the right ventricle (Fig. 5). The differential diagnosis included myxoma, organized thrombus, sarcoma, lymphoma, and metastatic lesions. We initiated Heparin infusion under the tentative diagnosis of intracardiac thrombosis, and surgical intervention was planned for the next day. However, dyspnea and hypoxemia progressed rapidly while the surgeon was discussing the need for intervention with the family. Due to respiratory failure and lactate acidosis, we initiated tracheal intubation, inotropes, and resuscitation 46 hours after admission. Respiratory decompensation with severe metabolic acidosis and multiple organ failure progressed despite medical treatment, and the patient expired 60 hours after admission.

\section{Discussion}

Cardiac mass is an unusual condition that can manifest as a variety of clinical symptoms. We report a case of a 72-yearold woman who presented with rapidly progressing right atrial mass extending into the right ventricle, inferior vena cava, and 


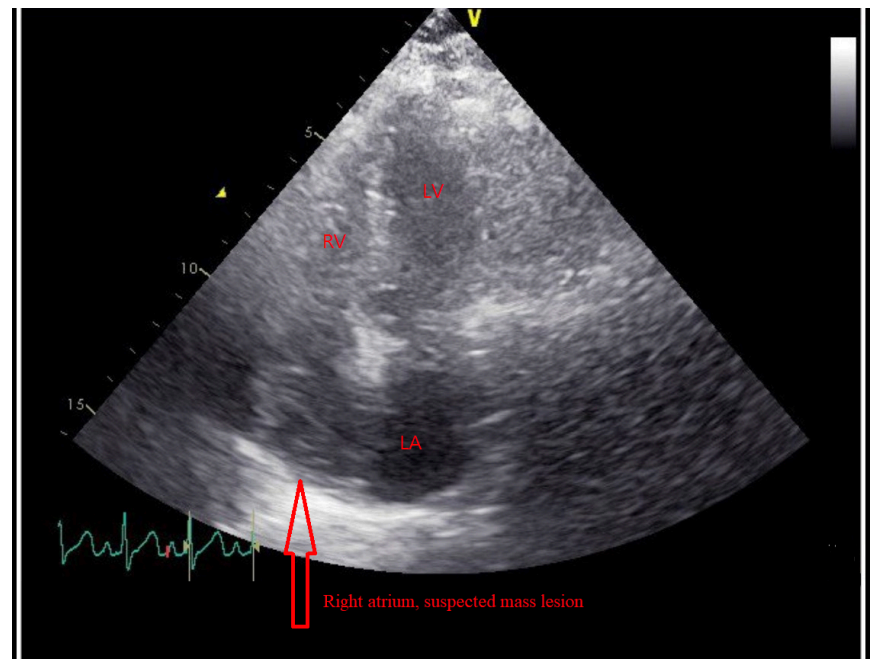

F I G URE 1. Transthoracic echocardiography, four chamber view.

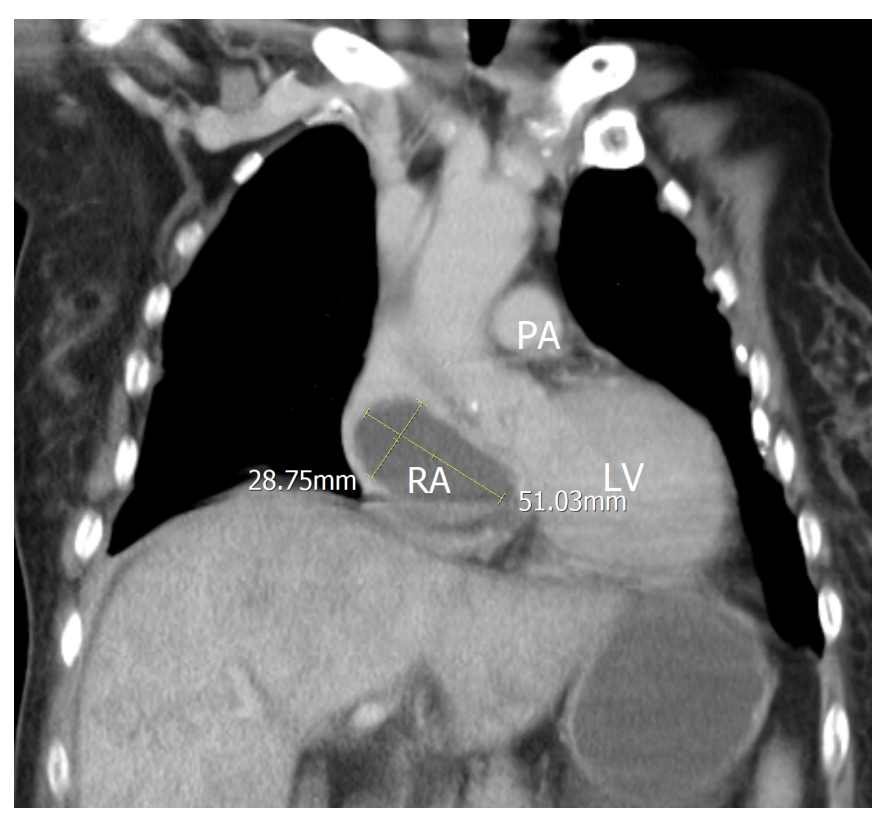

F I G U R E 2. Coronal section revealed a lesion about $67 \times$ $50 \times 63 \mathrm{~mm}$ in the right atrium.

PA, pulmonary artery; RA, right atrium; LV, left ventricle.

hepatic vein. Atrial masses can be classified into two types: cardiac thrombus and non-thrombus, i.e., tumors. Myxoma usually originates in the left atrium and is the most common primary cardiac tumor. Masses in the right atrium usually originate from metastatic malignancies.

On CT imaging, myxomas usually appear as round masses with a stem, floating randomly or even extending into the ventricle during the cardiac cycle. Atrial thrombosis usually occurs in patients with atrial fibrillation, structural heart disease, or those having an intra-cardiac device, most of which lack stems [1]. However, the differences between myxoma and thrombosis are difficult to distinguish from cardiac ultrasound or computer tomography. This patient did not exhibit any contributing factors, such as atrial fibrillation, structural heart disease, myocardial infarction, or previous heart failure, which indicate a high tendency to form cardiac thrombosis [2]. Other

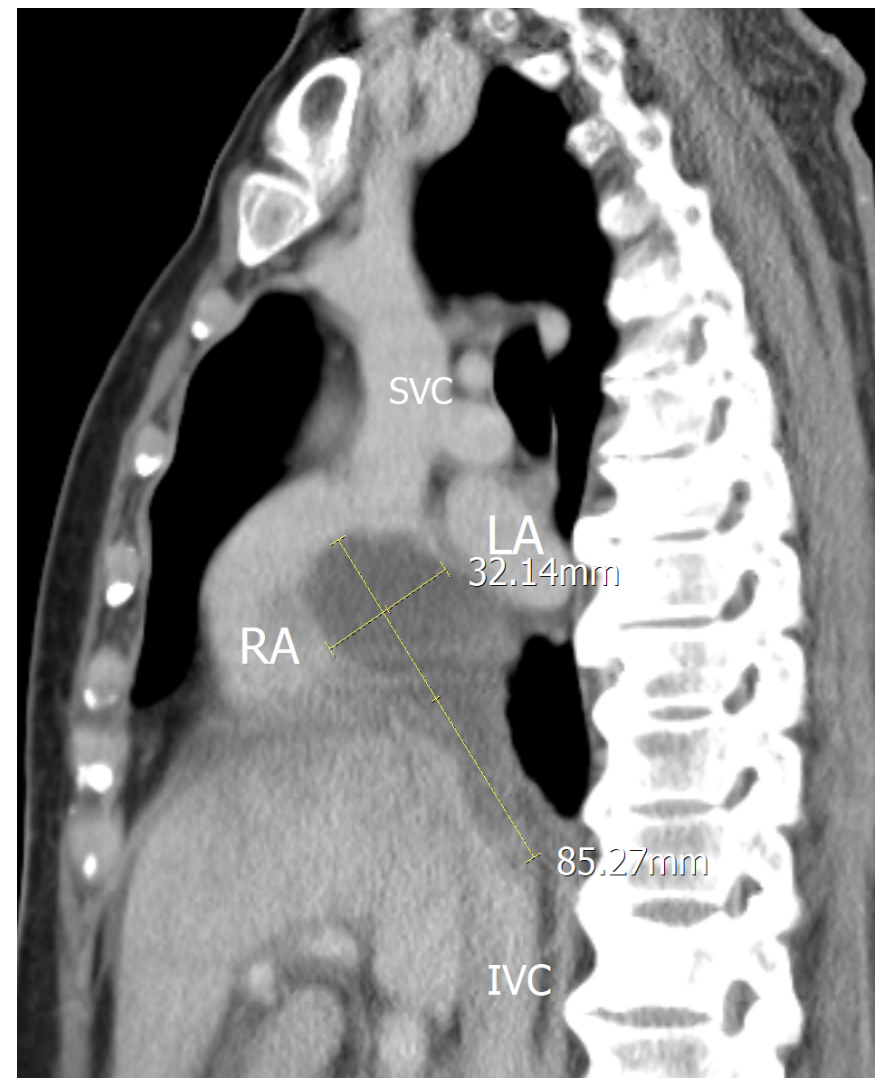

F I GURE 3. Sagittal section revealed a poorly enhanced lesion in the right atrium with inferior vena cava extension. LA/RA, left and right atrium; SVC/IVC, superior/inferior vena cava.

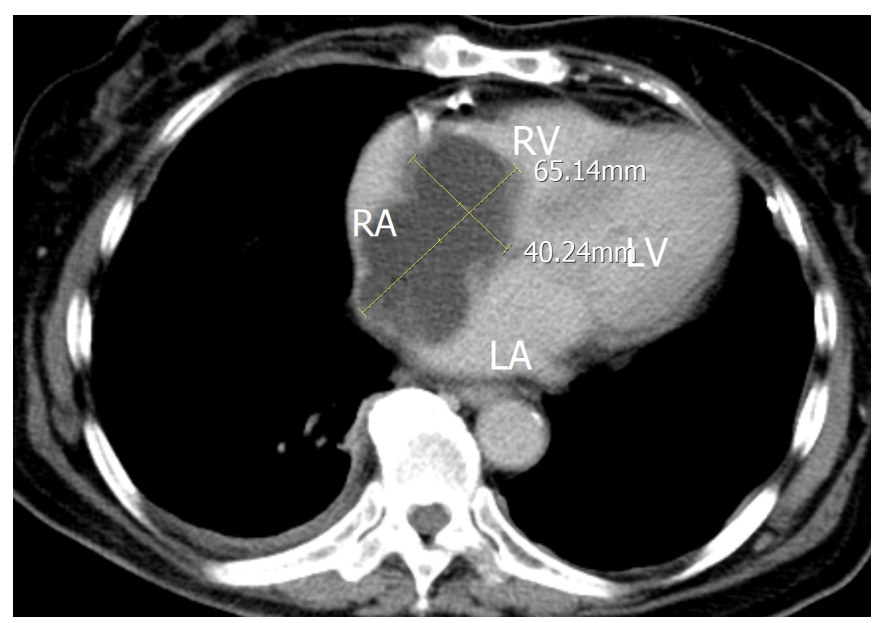

F I GURE 4. Axial section revealed a poorly enhanced lesion in the right atrium with inferior vena cava extension.

suggested causes of venous thrombosis include implantation of venous access devices, congenital coagulopathies, Behçet's disease, surgery, malignancy, trauma, pregnancy, obesity, long fly trip, and medication. Our patient did not have any of these signs and risks mentioned above. Torres and Doukky reported a massive obliterative right atrial thrombus in a 51year-old male who presented with a near-syncope. After undergoing a successful operation, the patient was discharged with Warfarin for a target international normalized ratio of 


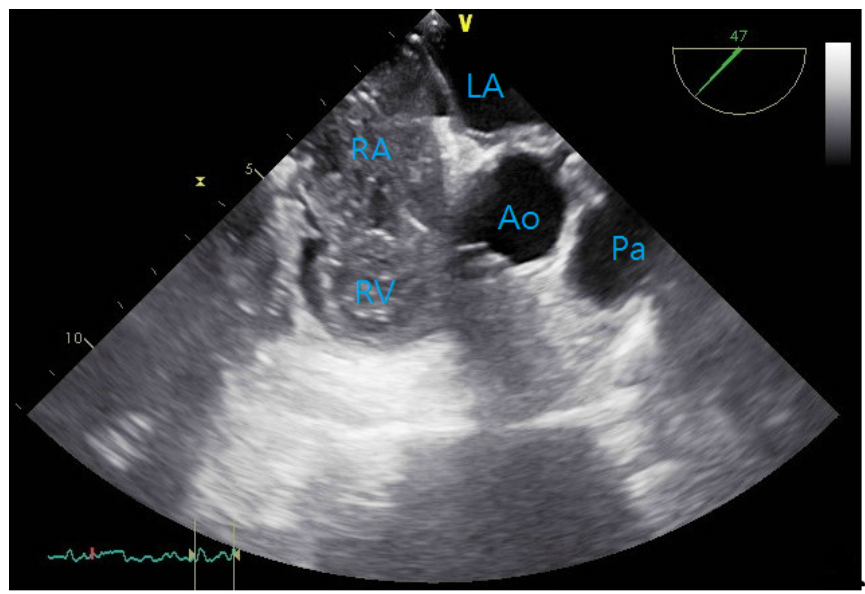

F I GURE 5. Transesophageal echocardiography, midesophageal.

Ao, aorta; Pa, pulmonary artery.

2.5-3.5 [3]. Cisico et al. [4] reported a calcified thrombosis originating from the right atrium and inferior vena cava. They recommended that surgical removal of the cardiac mass can help achieve both correct diagnosis and treatment to prevent further complications as embolic events or vessel occlusion. Based on the cases and diagnoses discussed so far, we believe that a detailed radiological study is required for diagnosis and surgical intervention. Using the above strategy could be the best course of action for patients diagnosed with intracardiac mass irrespective of the pathology.

Peripheral edema is a frequent symptom in patients with heart failure. Unusually, our patient presented no edema during her two visits to the ED. The mass, primarily located near the right atrium, might interrupt blood flow to the right heart than the left heart.

After reviewing the clinical course of our patient, we concluded that high quality imaging and image analysis is crucial for early diagnosis of cardiac mass. Diagnostic methods such as cardiac echography or contrast-enhanced image studies should have been performed during her first visit to allow for early diagnosis and resection. Several malignancies may show similar cardiac metastasis. However, due to the patient's critical condition, we did not have enough time to conduct more detailed diagnostic tests.

\section{AUTHOR CONTRIBUTIONS}

IHC and RCW designed the study and collect the information about this patient. IHC, LJL, and RCW conducted and analyzed the data. IHC, LJL wrote the original draft. RCW reviewed and revised the manuscript and supervised the study. All authors approved the final manuscript.

\section{ETHICS APPROVAL AND CONSENT TO PARTICIPATE}

This report was approved by Hualien Tzu Chi Hospital Research Ethics Committee at the moment (CR110-05). Written informed consent was obtained from the son of this patient for the patient's anonymized information to be published in this article. Data and images were obtained in accordance with the principles outlined in the Declaration of Helsinki.

\section{ACKNOWLEDGMENT}

The authors want to thank the family of this patient to agree us to present the condition of this patient, we also thank to Dr. Pau-Yuan Chang for his kindness and enthusiasm on teaching in radiology.

\section{FUNDING}

This research received no specific grant from any funding agency in the public, commercial, or not-for-profit sectors.

\section{CONFLICT OF INTEREST}

The authors declare no conflict of interest.

\section{HUMAN RIGHTS}

Our work does not infringe on any rights of others, including privacy rights, and intellectual property rights. There is no human rights violation in our manuscript.

\section{REFERENCES}

[1] Yang Y, Gu Z. A rare myxoma-like right atrial thrombus causing syncope. Medicine. 2018; 97: e12546.

[2] Javanshir E, Sadat-Ebrahimi S, Parvizi R, Toufan M, Sate H. Giant mass but small symptoms; huge thrombosis in the right atrium originating from the superior vena cava and protruding to the right ventricle: a case report. Journal of Medical Case Reports. 2019; 13: 312.

[3] Torres C, Doukky R. Massive obliterative right heart thrombus presenting with near-syncope. Echocardiography. 2019; 36: 1596-1597.

[4] Cisico S, Vacirca SR, Basso C, Mangino D. A rare case of a cardiac calcified thrombus originating from right atrium and inferior vena cava. Journal of Cardiac Surgery. 2020; 35: 703-705.

How to cite this article: I-Hsin Chang, Lian-Jie Lin, Ren$\mathrm{Chieh} \mathrm{Wu}$. Acute heart failure from a rapidly progressing large tumor in the right atrium. Signa Vitae. 2022;18(1):136-138. doi:10.22514/sv.2021.095 